\title{
Lidocaine Binding Enhances Inhibition of Nav1.7 Channels by the Sulfonamide PF-05089771
}

\author{
Sooyeon Jo and Bruce P. Bean \\ Department of Neurobiology, Harvard Medical School, Boston, Massachusetts
}

Received September 25, 2019; accepted February 27, 2020

\section{ABSTRACT}

PF-05089771 is an aryl sulfonamide Nav1.7 channel blocker that binds to the inactivated state of Nav1.7 channels with high affinity but binds only weakly to channels in the resting state. Such aryl sulfonamide Nav1.7 channel blockers bind to the extracellular surface of the S1-S4 voltage-sensor segment of homologous Domain 4, whose movement is associated with inactivation. This binding site is different from that of classic sodium channel inhibitors like lidocaine, which also bind with higher affinity to the inactivated state than the resting state but bind at a site within the pore of the channel. The common dependence on gating state with distinct binding sites raises the possibility that inhibition by aryl sulfonamides and by classic local anesthetics might show an interaction mediated by their mutual state dependence. We tested this possibility by examining the state-dependent inhibition by PF-05089771 and lidocaine of human Nav1.7 channels expressed in human embryonic kidney 293 cells. At $-80 \mathrm{mV}$, where a small fraction of channels are in an inactivated state under drug-free conditions, inhibition by PF-05089771 was both enhanced and speeded in the presence of lidocaine. The results suggest that lidocaine binding to the channel enhances PF-05089771 inhibition by altering the equilibrium between resting states (with D4S4 in the inner position) and inactivated states (with D4S4 in the outer position). The gating state-mediated interaction between the compounds illustrates a principle applicable to many state-dependent agents.

\section{SIGNIFICANCE STATEMENT}

The results show that lidocaine enhances the degree and rate of inhibition of Nav1.7 channels by the aryl sulfonamide compound PF-05089771, consistent with state-dependent binding by lidocaine increasing the fraction of channels presenting a highaffinity binding site for PF-05089771 and suggesting that combinations of agents targeted to the pore-region binding site of lidocaine and the external binding site of aryl sulfonamides may have synergistic actions.

\section{Introduction}

Local anesthetics, like lidocaine, act by inhibiting the voltage-gated sodium channels that generate action potentials. The binding site for local anesthetics is inside the poreforming region of the channel, and a wide variety of other pharmacological agents including antiepileptic drugs like carbamazepine bind to the same site [Ragsdale et al., 1994, 1996; Kuo, 1998; Yarov-Yarovoy et al., 2001, 2002; reviewed by Catterall (1999), Catterall and Swanson (2015)]. A common feature of these agents is that they bind with much higher affinity to the open and inactivated states of the channel induced by depolarization than to the resting state that predominates at normal resting potentials (Hille, 1977; Catterall, 1999). The high-affinity binding site for these drugs is apparently formed when the gating charge-containing S4 segments of the channel move from their more internal (resting) to more external (activated) positions (Vedantham and Cannon, 1999; Sheets and Hanck, 2007; Fozzard et al., 2011). These $\mathrm{S} 4$ movements promote inactivation (Kuo and Bean, 1994; Capes et al., 2013; Ahern et al., 2016), so the

This work was supported by the National Institutes of Health National Institute of Neurologic Diseases and Stroke [Grants NS036855 and NS110860] and by Pfizer (Neusentis).

https://doi.org/10.1124/mol.119.118380. formation of the high-affinity binding site for drugs like lidocaine roughly parallels the development of inactivation. However, it is probably the outward position of the S4 regions, especially those of the third and fourth pseudosubunits, that is important for high-affinity binding of local anesthetics rather than inactivation per se (Wang et al., 2004; Muroi and Chanda, 2009; Nguyen et al., 2019).

Recently, a new class of small-molecule inhibitors has been identified that interacts with the sodium channel in a completely different manner (McCormack et al., 2013; Bagal et al., 2014; Alexandrou et al., 2016; Focken et al., 2016, 2018; Flinspach et al., 2017; Pero et al., 2017; Wu et al., 2017, 2018). These molecules, based on an aryl sulfonamide scaffold, bind to the voltage-sensor region of the fourth pseudosubunit domain (VSD4) at a site that is on the external side of the plasma membrane (McCormack et al., 2013; Ahuja et al., 2015). Like local anesthetics, binding is strongly statedependent, with tight binding to inactivated channels and weak binding to resting channels (Alexandrou et al., 2016; Theile et al., 2016). A plausible model is that when the S4 region of VSD4 moves outward during inactivation (Capes et al., 2013; Hsu et al., 2017), it forms a high-affinity binding site for the aryl sulfonamide compounds (Ahuja et al., 2015). The state dependence of such agents may be important for 
their potential clinical efficacy and for designing screens for new compounds (Chernov-Rogan et al., 2018).

If binding of aryl sulfonamide compounds and classic sodium channel inhibitors occurs at different sites, and binding of one agent does not interfere with binding of another, there could be a mutual synergistic enhancement of channel inhibition by the two compounds because at any given voltage, binding of each compound can occur not only to normal drug-free inactivated channels but also to the new fraction of inactivated channels occupied by the other compound. On the other hand, binding of one compound might affect binding of the other. For example, binding of lidocaine to its site within the pore of the inactivated channel might alter the position of the VSD4 in such a way as to alter the binding site for the aryl sulfonamide compounds. To explore these possibilities, we tested whether binding of lidocaine to human Nav1.7 channels modifies the state-dependent interaction of the aryl sulfonamide compound PF-05089771. We found that the presence of lidocaine enhanced both the degree and speed of inhibition by PF-05089771, suggesting that statedependent binding by lidocaine results in an increased fraction of channels presenting a high-affinity binding site for PF-05089771.

\section{Materials and Methods}

Pharmacology. PF-05089771 (4-[2-(5-Amino-1H-pyrazol-4-yl)-4chloro-phenoxy]-5-chloro-2-fluoro- $N$-thiazol-4-yl-benzenesulfonamide; Swain et al., 2017) was obtained from Sigma-Aldrich as the p-toluene sulfonate salt (PZ0311; Sigma-Aldrich), prepared as a stock solution of $500 \mu \mathrm{M}$ in DMSO (Sigma), and diluted to final concentrations of 100-500 nM in recording solution. Lidocaine was purchased from Sigma-Aldrich and prepared as a stock solution of $100 \mathrm{mM}$ in distilled water.

Cell Culture. Human embryonic kidney 293 cells stably expressing human Nav1.7 channels (Liu et al., 2012) were grown in Eagle's Minimum Essential Medium (American Type Culture Collection) containing 10\% FBS (Sigma), $800 \mu \mathrm{g} / \mathrm{ml} \mathrm{G418} \mathrm{(Sigma),} \mathrm{and} \mathrm{penicil-}$ lin/streptomycin (Sigma) under $5 \% \mathrm{CO}_{2}$ at $37^{\circ} \mathrm{C}$.

Electrophysiology. Whole-cell recordings were obtained using patch pipettes with resistances of 2-3.5 $\mathrm{M} \Omega$ when filled with the internal solution consisting of $61 \mathrm{mM} \mathrm{CsF}, 61 \mathrm{mM} \mathrm{CsCl}, 9 \mathrm{mM} \mathrm{NaCl}$, $1.8 \mathrm{mM} \mathrm{MgCl} 2,9 \mathrm{mM}$ EGTA, $14 \mathrm{mM}$ creatine phosphate (Tris salt), $4 \mathrm{mM}$ MgATP, $0.3 \mathrm{mM}$ GTP (Tris salt), and $9 \mathrm{mM}$ HEPES, with $\mathrm{pH}$ adjusted to 7.2 with $\mathrm{CsOH}$. The shank of the electrode was wrapped with Parafilm to reduce capacitance and allow optimal series resistance compensation without oscillation. Seals were obtained and the whole-cell configuration was established with cells in Tyrode's solution containing $10 \mathrm{mM}$ TEACl ( $155 \mathrm{mM} \mathrm{NaCl}, 3.5 \mathrm{mM} \mathrm{KCl}, 10 \mathrm{mM}$

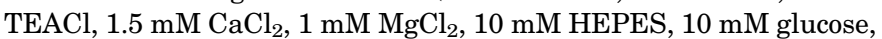
$\mathrm{pH}$ adjusted to 7.4 with $\mathrm{NaOH}$ ). After establishing whole-cell recording, cells were lifted off the bottom of the recording chamber and placed in front of an array of quartz flow pipes $(250 \mu \mathrm{m}$ internal diameter, $350 \mu \mathrm{m}$ external diameter). Recordings were made using the Tyrode's solution with $10 \mathrm{mM}$ TEACl. Solution changes were made (in $<1$ second) by moving the cell between adjacent pipes. Control solution contained the same DMSO concentration as the PF05089771-containing solution to which it was matched.

The amplifier was tuned for partial compensation of series resistance (typically $70 \%-80 \%$ of a total series resistance of 4-10 M $\Omega$ ), and tuning was periodically readjusted during the experiment. Currents were recorded at room temperature $\left(21-23^{\circ} \mathrm{C}\right)$ with an Axopatch 200 amplifier and filtered at $5 \mathrm{kHz}$ with a low-pass Bessel filter. Currents were digitized using a Digidata 1322A data acquisition interface controlled by pClamp9.2 software (Axon Instruments).
Analysis. Data were analyzed using programs written in Igor Pro 4.0 (Wavemetrics, Lake Oswego, OR) using DataAccess (Bruxton Software) to read pClamp data files into Igor Pro. Currents were corrected for linear capacitative and leak currents, which were determined using $5-\mathrm{mV}$ hyperpolarizations delivered from the resting potential (usually -100 or $-120 \mathrm{mV}$ ) and then appropriately scaled and subtracted. The time course of inhibition by PF-05089771 (or PF05089771 in $1 \mathrm{mM}$ lidocaine) could be fit well by a single exponential function; at concentrations of $200 \mathrm{nM}$ PF-05089771 or below, inhibition did not reach a steady state in 10 minutes, and the fitting allowed the asymptote to vary as a free parameter. Statistical analyses were performed using Igor Pro. Data are given as mean \pm S.D., and statistical significance was assessed with the two-tailed Mann-Whitney Test.

\section{Results}

We first determined the kinetics and state dependence of inhibition of human Nav1.7 channels under our recording conditions, using protocols similar to those introduced previously to study PF-05089771 inhibition (Alexandrou et al., 2016; Theile et al., 2016). Figure 1 shows the time course of dose-dependent inhibition by PF-05089771 using a pulse protocol in which binding of PF-05089771 is facilitated by long depolarizing prepulses. Sodium current is evoked by a 10millisecond depolarization to $0 \mathrm{mV}$ from a holding voltage of $120 \mathrm{mV}$. In each delivery of the pulse protocol, binding of PF05089771 is facilitated by a 4 -second prepulse to $0 \mathrm{mV}$, which puts channels into inactivated states with high affinity for PF05089771 (Alexandrou et al., 2016; Theile et al., 2016). After the prepulse, a 3 -second return to $-120 \mathrm{mV}$ enables nearly complete recovery from non-drug-bound channels, including slow inactivated channels (Jo and Bean, 2011, 2017), but is too short for any significant recovery from drug-bound inactivated states (Theile et al., 2016). Thus, this protocol (repeated every 15 seconds) effectively monitors the time course of drug binding. Drug binding occurs almost entirely during the prepulse to $0 \mathrm{mV}$, during which channels are in the inactivated state after the first few milliseconds, because there is essentially no inhibition if the protocol is run without a prepulse, thus delivering the 10-millisecond test pulse from a steady holding potential of $-120 \mathrm{mV}$ (Theile et al., 2016). With this protocol, a 10-minute application of $500 \mathrm{nM}$ PF-05089771 produced steady-state inhibition of $95 \% \pm 2 \%(n=6$, mean \pm S.D.). With lower concentrations of PF-05089771, inhibition was slower, and it was not feasible to determine a steady-state dose-response relationship at lower concentrations. Therefore, in designing subsequent experiments, we focused on the time course of inhibition. This was strongly concentrationdependent, with the time constant for inhibition by $100 \mathrm{nM}$ PF-05089771 (323 \pm 85 seconds, mean \pm S.D., $n=6)$ decreasing about 2-fold with $200 \mathrm{nM}$ PF-05089771 (151 \pm 12 seconds, $n=6$ ) and about 5 -fold with $500 \mathrm{nM} \mathrm{PF-05089771}$ (71 \pm 8 seconds, $n=6$ ). This relationship is roughly what is expected if PF-05089771 binds to inactivated channels during the prepulse with a binding rate constant proportional to PF05089771 concentration and a much slower unbinding rate constant.

The slow time course of inhibition by PF-05089771 seen with the protocol in Fig. 1, with only a small increment in inhibition with each application of a 4-second prepulse to $0 \mathrm{mV}$, raises the possibility that PF-05089771 occurs by binding to slow inactivated states of the channels, which are 
A
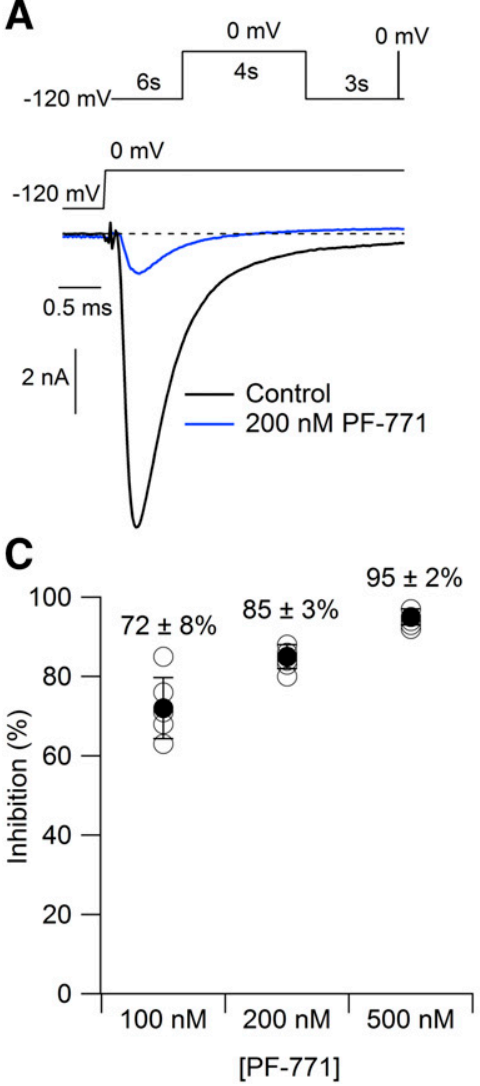
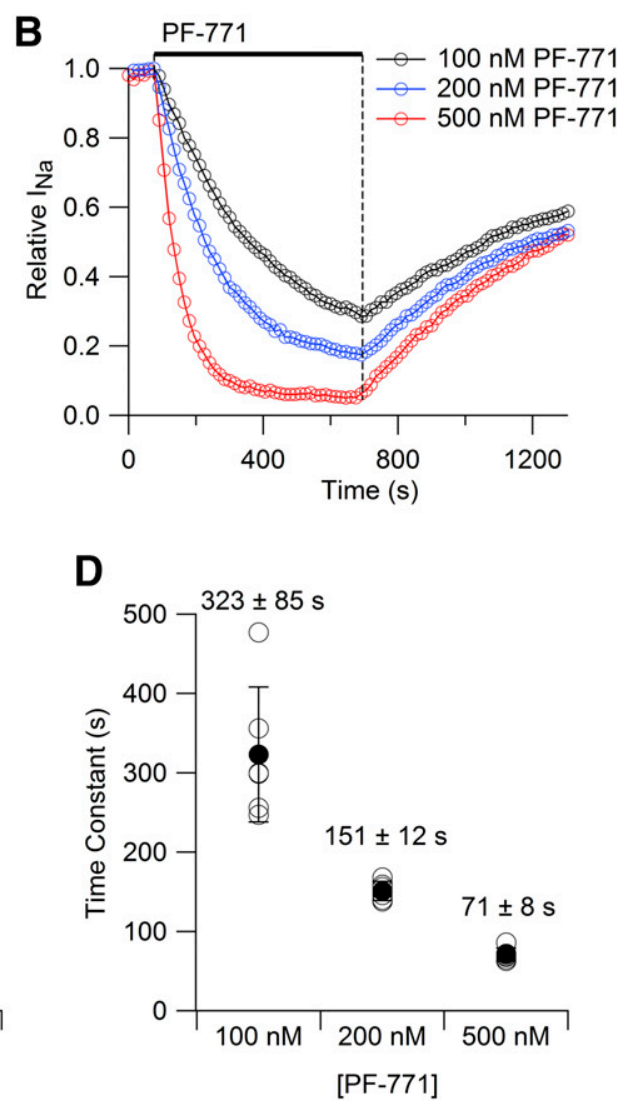

Fig. 1. PF-05089771 (PF-771) inhibition of human Nav1.7 channels. (A) Top: pulse protocol. Sodium current was assayed by a 10 -millisecond test pulse to $0 \mathrm{mV}$ from a holding potential of $-120 \mathrm{mV}$. Binding of PF05089771 was facilitated by a 4 -second prepulse depolarization to $0 \mathrm{mV}$, followed by a 3-second return to $120 \mathrm{mV}$ to allow recovery from inactivation of channels not bound to drug (including recovery from slow inactivation). The sequence was repeated every 15 seconds. Bottom: Currents evoked by the test pulse before and after exposure to $200 \mathrm{nM}$ PF-05089771 for 10 minutes. (B) Time course of sodium current $\left(\mathrm{I}_{\mathrm{Na}}\right)$ inhibition by 100,200 , and $500 \mathrm{nM}$ PF-05089771. (C) Collected results for inhibition produced by 10 minutes of drug exposure with 4 -second prepulses to $0 \mathrm{mV}$. Open circles show results from individual cells, and closed symbols show mean \pm S.D., $n=6$ for each concentration of PF-05089771. (D) Collected results for time constant of inhibition. Open circles show results from individual cells, and closed symbols show mean \pm S.D., $n=6$ for each concentration of PF-05089771. produced by long depolarizations of sodium channels. However, it can be difficult to distinguish selective binding to slow inactivated states from high-affinity binding to fast inactivated states that simply occurs with slow on- and off-rates (Karoly et al., 2010). Figure 2 shows the results with a protocol used previously to discriminate between these mechanisms (Jo and Bean, 2011, 2017) based on the different voltage dependence of occupancy of fast and slow inactivated states. The experiment compares the kinetics of drug inhibition at two different voltages: $-40 \mathrm{mV}$, where fast inactivation is complete (Fig. 2A, open circles) but slow inactivation is not, and at $0 \mathrm{mV}$, where slow inactivation is maximal (Fig. 2A, closed circles). If drug binding occurs selectively to slow inactivated states, the time course of inhibition should be much faster at $0 \mathrm{mV}$, where a substantial fraction of channels are in the slow inactivated state, than at $-40 \mathrm{mV}$, where few channels are in the slow inactivated state, and most channels are in the fast inactivated state. In fact, however, the time course of inhibition is nearly identical at the two voltages, occurring with a time constant of $167 \pm 31$ seconds (mean \pm S.D.) with prepulses to $-40 \mathrm{mV}(n=6)$ and $151 \pm 12$ seconds (mean \pm S.D.) with prepulses to $0 \mathrm{mV}(n=6)(P=0.68$, twotailed Mann-Whitney Test). As expected, with a prepulse to $80 \mathrm{mV}$, where a smaller fraction of channels are in any inactivated state, the time course of inhibition was slower (time constant of $284 \pm 52$ seconds, mean \pm S.D., $n=5)(0 \mathrm{mV}$ vs. $-80 \mathrm{mV}, P=0.008$, two-tailed Mann-Whitney Test). These results fit well with a previous study, wherein the inhibition by PF-05089771 was found to depend on the total time spent at depolarized voltages where channels are inactivated, regardless of whether the time is divided into many short prepulses, where channels are mostly in fast inactivated states, or fewer long prepulses, where channels are mainly in slow inactivated states (Theile et al., 2016).

Next, we examined whether channel occupancy by lidocaine affects binding of PF-05089771. First, we characterized the effects of lidocaine applied alone. We used a high concentration of lidocaine $(1 \mathrm{mM})$ because for these experiments, we wanted to explore the rate of PF-05089771 binding to channels that are all occupied by lidocaine. At $1 \mathrm{mM}$, lidocaine inhibited current evoked from a holding potential of $-120 \mathrm{mV}$ by $31 \% \pm$ $8 \%$ (mean \pm S.D., $n=4$, consistent with a $\mathrm{K}_{\mathrm{d}}$ of binding of $2.2 \mathrm{mM}$ to the low-affinity resting state of the channel) (see Bean et al., 1983). With a holding potential of $-80 \mathrm{mV}$, where channels were partially inactivated (on avg. by $20 \% \pm 9 \%, n=$ 22), $1 \mathrm{mM}$ lidocaine inhibited current evoked by a step to $0 \mathrm{mV}$ completely (Fig. 3A), consistent with far higher affinity to inactivated channels. After complete inhibition at $-80 \mathrm{mV}$, when the membrane voltage was changed to $-120 \mathrm{mV}$, lidocaine-bound channels recovered availability in about a second (Fig. 3B). Figure 3C shows the time course of inhibition by $1 \mathrm{mM}$ lidocaine using a pulse protocol incorporating 4 -second conditioning pulses to $-80 \mathrm{mV}$ (which would produce essentially complete occupancy of sodium channels by lidocaine) followed by 3 seconds at $-120 \mathrm{mV}$ (long enough to completely remove the extra inhibition produced by lidocaine binding at $-80 \mathrm{mV}$ ) and then a test pulse to $0 \mathrm{mV}$. With this protocol, lidocaine inhibited test pulse current by $30 \% \pm$ 9\% (mean \pm S.D., $n=23$ ) and, with the pulse protocol delivered every 15 seconds, inhibition by lidocaine developed and recovered within one cycle. Thus, this protocol is ideal for testing whether lidocaine binding can enhance binding of 

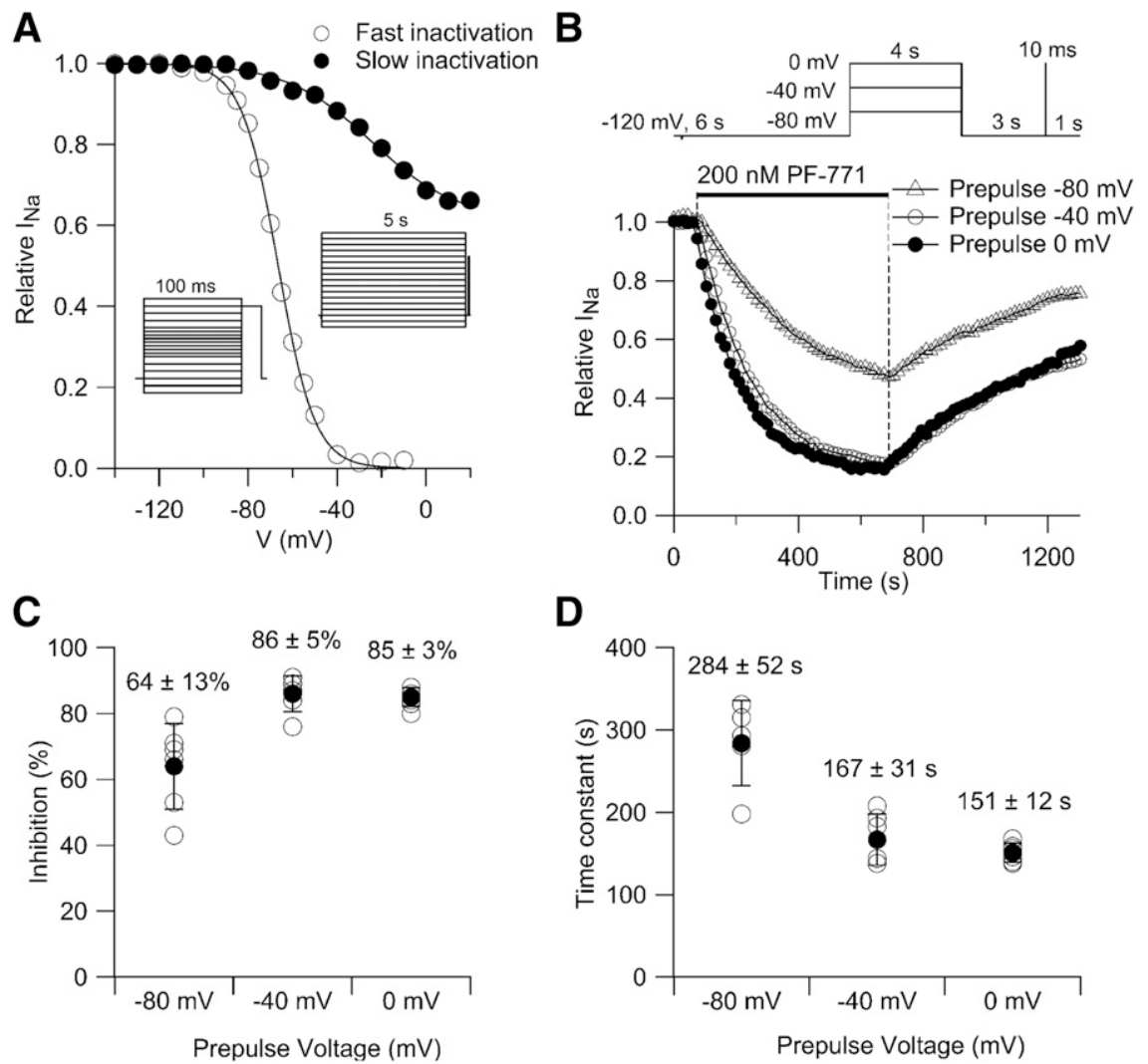

Fig. 2. Dependence of PF-05089771 inhibition on prepulse voltage. (A) Voltage dependence of occupancy of fast and slow inactivated states in absence of drug. Occupancy of fast inactivated states (open circles) was assayed by sodium current $\left(\mathrm{I}_{\mathrm{Na}}\right)$ evoked by a 20 -millisecond test step to $-20 \mathrm{mV}$ after 100 millisecond prepulses to varying voltages normalized to the current evoked from $-140 \mathrm{mV}$. Solid curve: best fit by the Boltzmann Eq. $1 /\left(1+\exp \left(\left(V_{m}-\right.\right.\right.$ $\left.\left.V_{h}\right) / k\right)$ ), wherein $V_{m}$ is conditioning potential, $V_{h}$ is voltage of half-maximal inactivation, and $k$ is the slope factor, with $V_{\mathrm{h}}=-66.5 \mathrm{mV}$ and $k=8.2$; collected values $V_{\mathrm{h}}=-65 \pm 8 \mathrm{mV}$, and $k=8 \pm 2.4 \mathrm{mV}$ (mean \pm S.D., $n=23$ ). Slow inactivation (filled circles) was measured with a 5-second depolarization to varying voltages, returning to $-120 \mathrm{mV}$ for 100 milliseconds to allow recovery from fast inactivation and then assaying channel availability with a test pulse to $-20 \mathrm{mV}$. Relative sodium current was normalized to that with a conditioning pulse to $-140 \mathrm{mV}$. Data are fit by a modified Boltzmann equation (Carr et al., 2003): $I / I_{\max }=\left(1-I_{\text {resid }}\right) /\left(\left(1+\exp \left(\left(V_{\mathrm{m}}-V_{\mathrm{h}}\right) / k\right)\right)+I_{\text {resid }}\right.$, with $V_{\mathrm{h}}=-31 \pm 9 \mathrm{mV}, k=16 \pm 6 \mathrm{mV}$, and $I_{\text {resid }}=0.7 \pm 0.2$ (mean \pm S.D., $n=8$ ). (B) Time course of inhibition by $200 \mathrm{nM}$ PF-05089771 using stimulation prototcols as in Fig. 1 with 4 -second prepulses to $-80,-40$, or $0 \mathrm{mV}$. (C) Collected results for inhibition produced by 10 minutes of drug exposure with protocols using prepulses to each voltage. Open circles show results from individual cells, and closed symbols show mean \pm S.D., $n=6$ for each prepulse voltage. (D) Collected results for time course of inhibition with prepulses to each voltage. Open circles show results from individual cells, and closed symbols show mean \pm S.D., $n=6$ for each prepulse voltage. The time constant for best fit of a single exponential decay to steady state, with prepulses to $-80 \mathrm{mV}(284 \pm 52$ seconds, $n=5),-40 \mathrm{mV}$ (167 $\pm 31 \mathrm{~seconds}, n=6$ ), or $0 \mathrm{mV}(151 \pm 12$ seconds, $n=6)(0 \mathrm{mV}$ vs. $-40 \mathrm{mV}, P=0.68 ; 0 \mathrm{mV}$ vs. $-80 \mathrm{mV}, P=0.008$, two-tailed Mann-Whitney Test $)$. Open circles indicate individual experiments, and filled circles show mean \pm S.D. from collected results.

PF-05089771 because binding of PF-05089771 during the protocol occurs during the 4-second depolarization to $-80 \mathrm{mV}$, where channels are all occupied by lidocaine (if $1 \mathrm{mM}$ lidocaine is present), but although lidocaine unbinds rapidly during the following 3-second period at $-120 \mathrm{mV}, \mathrm{PF}-05089771$ does not, so repeated cycles of the pulse protocol can monitor the rate and extent of PF-05089771 binding.

Figure 4 shows the results of experiments using this pulse protocol to test whether channel occupancy by lidocaine affects binding of PF-05089771. These experiments showed that exposure to lidocaine enhanced channel inhibition by $\mathrm{PF}$ 05089771. Applied for 10 minutes, $100 \mathrm{nM}$ PF-05089771 applied alone inhibited by $51 \% \pm 6 \%$ (mean \pm S.D., $n=7$ ), whereas $100 \mathrm{nM}$ PF-05089771 applied in the presence of $1 \mathrm{mM}$ lidocaine inhibited by $83 \% \pm 7 \%$ (normalizing inhibition to the current in $1 \mathrm{mM}$ lidocaine alone just before application of 100 nM PF-05089771; $n=9 ; P=0.001$, two-tailed Mann-Whitney Test). Also, as expected if a larger fraction of channels present a high-affinity binding site for PF-05089771 in the presence of lidocaine (a new fraction of inactivated channels bound to lidocaine as well as inactivated channels not bound) than without (only the fraction of inactivated channels), the development of PF-05089771 inhibition was faster when in the presence of lidocaine (time constant of $184 \pm 37$ seconds, mean \pm S.D., $n=9$ ) than in the absence of lidocaine (time constant of $444 \pm 117$ seconds, mean \pm S.D., $n=6)(P=0.0018$, two-tailed Mann-Whitney Test).

\section{Discussion}

Synergistic Inhibition by Lidocaine and PF-05089771. These results show that inhibition by PF-05089771 is enhanced in the presence of lidocaine, suggesting a synergistic action of the two types of inhibitors. The most likely mechanism for this effect is that both agents interact with particularly high affinity with channels in which the voltage-sensor regions of the channels are in the more external "activated" position yet bind at distinct sites. Thus, the mass-action effect of each inhibitor to "pull" channels into conformations with voltage sensors in the external position results in a larger 
A
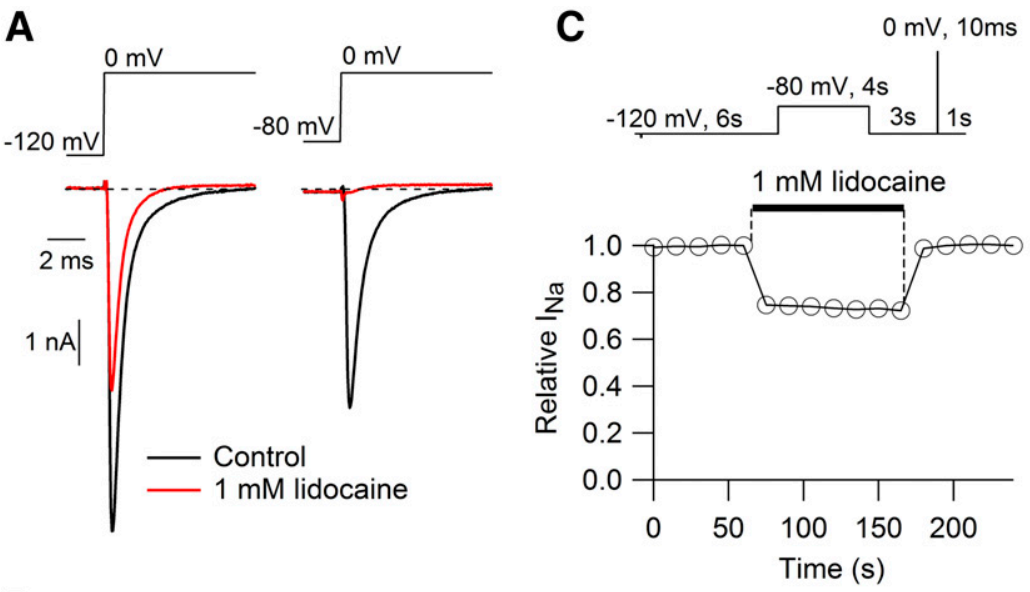

B

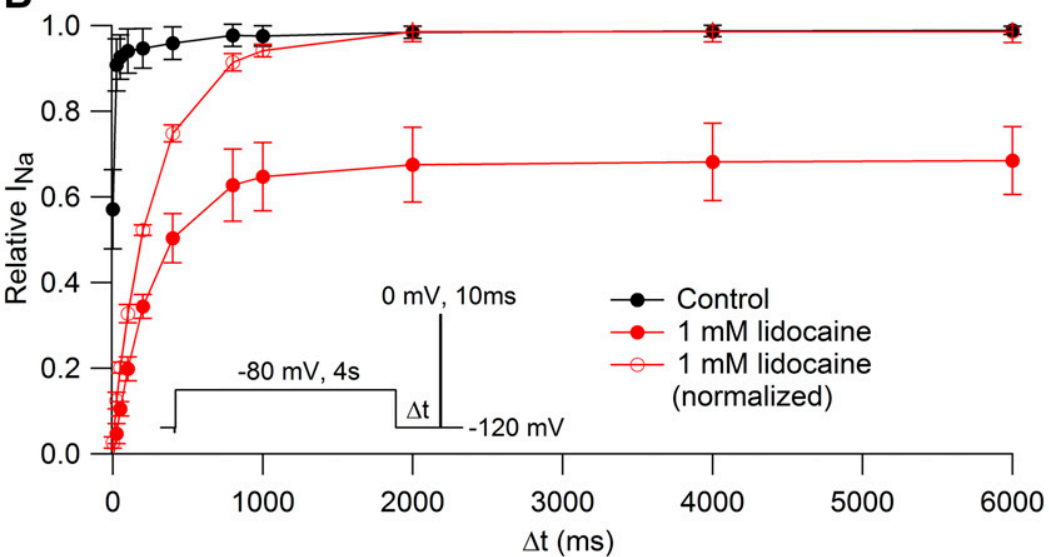

Fig. 3. Lidocaine binding to Nav1.7 channels is complete at $-80 \mathrm{mV}$ and recovers in 3 seconds at $-120 \mathrm{mV}$. (A) Effect of $1 \mathrm{mM}$ lidocaine on current evoked at $0 \mathrm{mV}$ when applied from a holding potential of $-120 \mathrm{mV}$ or after holding at $80 \mathrm{mV}$ for 4 seconds. (B) Time course of recovery of sodium channels at $-120 \mathrm{mV}$ after a 4 -second conditioning pulse to $-80 \mathrm{mV}$. Black filled circles: sodium current $\left(\mathrm{I}_{\mathrm{Na}}\right)$ in control, normalized to maximal current (measured after 10 seconds at $-120 \mathrm{mV}$ ). Red filled circles: currents in $1 \mathrm{mM}$ lidocaine, normalized to maximal control current. Red open circles: currents in $1 \mathrm{mM}$ lidocaine, normalized to maximal current in lidocaine. Mean \pm S.D., $n=4$. The pulse protocol was repeated every 15 seconds with increasing $\Delta$ t up to 10 seconds. (C) Time course of inhibition and recovery by $1 \mathrm{mM}$ lidocaine applied with illustrated pulse protocol. fraction of all channels with voltage sensors in the external position, mimicking the effect of a more depolarized resting potential and increasing the fraction of channels presenting a high-affinity site for binding of the other inhibitor at its separate site. Channels with voltage sensors in the external, "activated" position are in inactivated states except for the first few milliseconds after a voltage step, during which they pass transiently through open states. Because binding of both lidocaine and PF-05089771 occurs over hundreds of millseconds to many seconds, binding of both agents will occur primarily to inactivated states. Thus, it is reasonable to view both agents as stabilizing inactivated states of the channel, even though it is movement of the voltage-sensor regions from more internal to more external "activated" positions rather than inactivation per se that results in formation of the distinct high-affinity binding sites for both lidocaine (Vedantham and Cannon, 1999; Wang et al., 2004; Sheets and Hanck, 2007; Fozzard et al., 2011) and PF-05089771 (Ahuja et al., 2015).

Contrast with Lacosamide and Lidocaine. Previously, we have done similar experiments examining interaction between sodium channel inhibition by lidocaine and lacosamide (Jo and Bean, 2017). These showed the opposite effect: the extent of lacosamide inhibition was reduced in the presence of lidocaine, which was consistent with binding of lidocaine preventing binding of lacosamide, fitting with other evidence that the two agents bind at the same binding site (Wang and Wang, 2014).

Tight Binding of PF-05089771 to Both Fast and Slow Inactivated States. Together with a previous study (Theile et al., 2016), the results in Fig. 2 showing that the extent and kinetics of PF-05089771 inhibition are almost identical at voltages of $-40 \mathrm{mV}$ (where fast inactivation is complete but there is little slow inactivation) and $0 \mathrm{mV}$ (where slow inactivation is nearly maximal) suggest that PF-05089771 binds equally well to fast and slow inactivated states. This is consistent with the expectation that the voltage-sensor regions of the channel would be in the external position in both fast and slow inactivated states. Interestingly, lidocaine binding to Nav1.7 channels may be different in this respect because lidocaine binding appears to hinder entry of channels into the slow inactivated state (Sheets et al., 2011). The structural basis of this effect is unknown and may be specific to Nav1.7 channels. Under physiologic or even pathophysiological conditions, the voltage range over which lidocaine binding and PF-05089771 binding might interact is unlikely to be more positive than $-40 \mathrm{mV}$ and so might be expected to involve primarily binding to fast inactivated states.

Clinical Implications. It remains to be seen whether the synergistic sodium channel inhibition by lidocaine and PF-05089771 can be exploited clinically. In vivo, the efficacy and time course of both injected and orally administered pain inhibitors depend strongly on their pharmacokinetics in addition to their fundamental action on sodium channels (e.g., Sun et al., 2019). Our results would predict that local injection of lidocaine and PF-05089771 would synergistically enhance nerve block compared with either agent alone. Interestingly, such synergy was recently reported for bupivacaine and neosaxitoxin (Lobo et al., 2015; Templin et al., 2015); in this case, a synergistic action at the level of channel block 
A
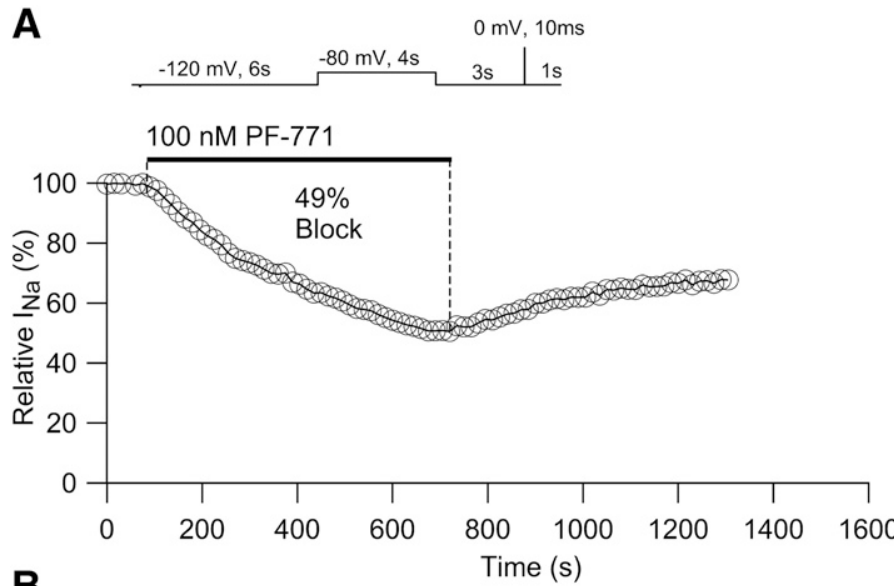

B

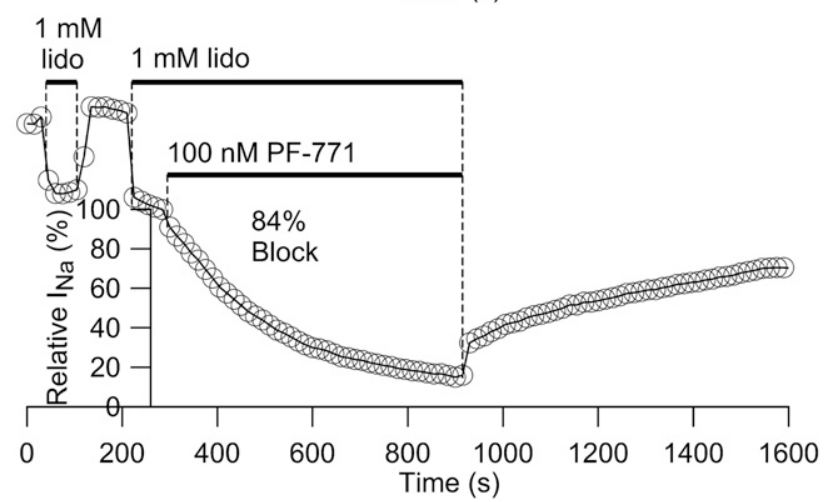

C
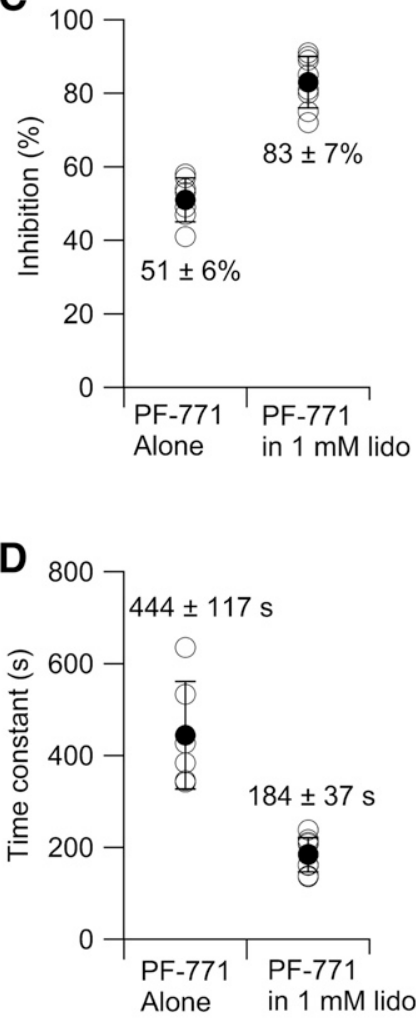

Fig. 4. Inhibition by PF-05089771 is enhanced and accelerated by lidocaine binding. (A) Top: pulse protocol. Sodium current ( $\mathrm{I}_{\mathrm{Na}}$ ) was assayed by a 10 millisecond test pulse to $0 \mathrm{mV}$, with binding of PF-05089771 and lidocaine facilitated by a 4-second prepulse depolarization to $-80 \mathrm{mV}$, which was followed by a 3-second return to $-120 \mathrm{mV}$ to allow recovery of channels that are not bound to PF-05089771. The sequence was repeated every 15 seconds. (A) Bottom: block by $100 \mathrm{nM}$ PF-05089771 in the absence of lidocaine. (B) Block by $100 \mathrm{nM}$ PF-05089771 in the presence of $1 \mathrm{mM}$ lidocaine, normalized to the current in lidocaine alone. (C) Collected results for inhibition by $100 \mathrm{nM} \mathrm{PF}-05089771$ in the absence $(51 \% \pm 6 \%, n=7)$ or presence $(83 \% \pm 7 \%, n=9)$ of $1 \mathrm{mM}$ lidocaine $(P=0.001$, two-tailed Mann-Whitney Test). (D) Tau for inhibition by $100 \mathrm{nM} \mathrm{PF}-05089771$ in the absence $(444 \pm 117$ seconds, $n=6)$ or presence (184 \pm 37 seconds, $n=9)$ of $1 \mathrm{mM}$ lidocaine $(P=0.0018$, two-tailed Mann-Whitney Test). Open circles indicate individual experiments, and filled circles indicate mean \pm S.D. from collected results.

has not yet been investigated but is plausible because binding of tetrodotoxin (closely related to saxitoxin) favors the outward position of the domain IV voltage sensor (Capes et al., 2012), producing a component of use-dependent inhibition (Patton and Goldin, 1991). However, the state dependence of tetrodotoxin or saxitoxin binding is much weaker than that of PF-05089771, suggesting that synergy between PF-05089771 and local anesthetics is likely stronger.

The action of lidocaine to enhance inhibition by PF05089771 is expected to be shared by a wide range of clinically used sodium channel inhibitors that bind at the same site as lidocaine and similarly stabilize inactivated states of the channel. Such agents include other local anesthetics; antiepileptic drugs like carbamazepine, phenytoin, lamotrigine, and lacosamide; and probably amitriptyline, an antidepressant also used to treat neuropathic pain, in which it likely acts in part by sodium channel inhibition (Kalso, 2005; Dick et al., 2007). Although studies have shown utility of the new Nav1.7 aryl sulfonamide compounds for treating pain in various animal models (Focken et al., 2016; Flinspach et al., 2017; Pero et al., 2017; Wu et al., 2017; Sun et al., 2019), it is still unclear how effective they will be in humans and for what conditions. Because of the unusual anionic nature of the compounds associated with the negatively charged "warhead," it is possible that tissue distribution may present unusual challenges and limit the concentration of compound that can be achieved at nerve endings or axons. The very slow binding of the compounds to channels even in the inactivated state may also limit the fraction of channels inhibited. Our results suggest that combination therapies with agents like orally active phenytoin, carbamazepine, or amitriptyline may synergistically enhance the efficacy of the compounds for treating pain systemically.

\section{Acknowledgments}

We are grateful to Mark Chapman, Neil Castle, Jonathan Theile, and Douglas Krafte for helpful discussions and suggestions.

\section{Authorship Contributions}

Participated in research design: Jo, Bean.

Conducted experiments: Jo.

Performed data analysis: Jo, Bean.

Wrote or contributed to the writing of the manuscript: Jo, Bean.

\section{References}

Ahern CA, Payandeh J, Bosmans F, and Chanda B (2016) The hitchhiker's guide to the voltage-gated sodium channel galaxy. J Gen Physiol 147:1-24.

Ahuja S, Mukund S, Deng L, Khakh K, Chang E, Ho H, Shriver S, Young C, Lin S, Johnson JP Jr., et al. (2015) Structural basis of Nav1.7 inhibition by an isoformselective small-molecule antagonist. Science 350:aac5464.

Alexandrou AJ, Brown AR, Chapman ML, Estacion M, Turner J, Mis MA, Wilbrey A Payne EC, Gutteridge A, Cox PJ, et al. (2016) Subtype-selective small molecule 
inhibitors reveal a fundamental role for Nav1.7 in nociceptor electrogenesis, axonal conduction and presynaptic release. PLoS One 11:e152405.

Bagal SK, Chapman ML, Marron BE, Prime R, Storer RI, and Swain NA (2014) Recent progress in sodium channel modulators for pain. Bioorg Med Chem Lett 24: $3690-3699$

Bean BP, Cohen CJ, and Tsien RW (1983) Lidocaine block of cardiac sodium channels. J Gen Physiol 81:613-642.

Capes DL, Arcisio-Miranda M, Jarecki BW, French RJ, and Chanda B (2012) Gating transitions in the selectivity filter region of a sodium channel are coupled to the domain IV voltage sensor. Proc Natl Acad Sci USA 109:2648-2653.

Capes DL, Goldschen-Ohm MP, Arcisio-Miranda M, Bezanilla F, and Chanda B (2013) Domain IV voltage-sensor movement is both sufficient and rate limiting for fast inactivation in sodium channels. J Gen Physiol 142:101-112.

Carr DB, Day M, Cantrell AR, Held J, Scheuer T, Catterall WA, and Surmeier DJ (2003) Transmitter modulation of slow, activity-dependent alterations in sodium channel availability endows neurons with a novel form of cellular plasticity. $\mathrm{Neu}$ ron 39:793-806.

Catterall WA (1999) Molecular properties of brain sodium channels: an important target for anticonvulsant drugs. Adv Neurol 79:441-456.

Catterall WA and Swanson TM (2015) Structural basis for pharmacology of voltagegated sodium and calcium channels. Mol Pharmacol 88:141-150.

Chernov-Rogan T, Li T, Lu G, Verschoof H, Khakh K, Jones SW, Beresini MH, Liu C, Ortwine DF, McKerrall SJ, et al. (2018) Mechanism-specific assay design facilitates the discovery of Nav1.7-selective inhibitors. Proc Natl Acad Sci USA 115: E792-E801.

Dick IE, Brochu RM, Purohit Y, Kaczorowski GJ, Martin WJ, and Priest BT (2007) Sodium channel blockade may contribute to the analgesic efficacy of antidepressants. J Pain 8:315-324.

Flinspach M, Xu Q, Piekarz AD, Fellows R, Hagan R, Gibbs A, Liu Y, Neff RA Freedman J, Eckert WA, et al. (2017) Insensitivity to pain induced by a potent selective closed-state Nav1.7 inhibitor. Sci Rep 7:39662.

Focken T, Chowdhury S, Zenova A, Grimwood ME, Chabot C, Sheng T, Hemeon I, Decker SM, Wilson M, Bichler P, et al. (2018) Design of conformationally constrained acyl sulfonamide isosteres: identification of $\mathrm{n}$ - $([1,2,4]$ triazolo[4,3- a]pyridin-3-yl)methane-sulfonamides as potent and selective hNav1.7 inhibitors for the treatment of pain. J Med Chem 61:4810-4831.

Focken T, Liu S, Chahal N, Dauphinais M, Grimwood ME, Chowdhury S, Hemeon I, Bichler P, Bogucki D, Waldbrook M, et al. (2016) Discovery of aryl sulfonamides as isoform-selective inhibitors of Nav1.7 with efficacy in rodent pain models. ACS Med Chem Lett 7:277-282.

Fozzard HA, Sheets MF, and Hanck DA (2011) The sodium channel as a target for local anesthetic drugs. Front Pharmacol 2:68.

Hille B (1977) Local anesthetics: hydrophilic and hydrophobic pathways for the drugreceptor reaction. J Gen Physiol 69:497-515.

Hsu EJ, Zhu W, Schubert AR, Voelker T, Varga Z, and Silva JR (2017) Regulation of $\mathrm{Na}^{+}$channel inactivation by the DIII and DIV voltage-sensing domains. J Gen Physiol 149:389-403.

Jo S and Bean BP (2011) Inhibition of neuronal voltage-gated sodium channels by brilliant blue G. Mol Pharmacol 80:247-257.

Jo S and Bean BP (2017) Lacosamide inhibition of Nav1.7 voltage-gated sodium channels: slow binding to fast-inactivated states. Mol Pharmacol 91:277-286.

Kalso E (2005) Sodium channel blockers in neuropathic pain. Curr Pharm Des 11: 3005-3011.

Karoly R, Lenkey N, Juhasz AO, Vizi ES, and Mike A (2010) Fast- or slow-inactivated state preference of $\mathrm{Na}+$ channel inhibitors: a simulation and experimental study. PLoS Comput Biol 6:e1000818.

Kuo CC (1998) A common anticonvulsant binding site for phenytoin, carbamazepine, and lamotrigine in neuronal Na+ channels. Mol Pharmacol 54:712-721.

Kuo CC and Bean BP (1994) Na+ channels must deactivate to recover from inactivation. Neuron 12:819-829.

Liu P, Jo S, and Bean BP (2012) Modulation of neuronal sodium channels by the sea anemone peptide BDS-I. J Neurophysiol 107:3155-3167.

Lobo K, Donado C, Cornelissen L, Kim J, Ortiz R, Peake RW, Kellogg M, Alexander ME, Zurakowski D, Kurgansky KE, et al. (2015) A phase 1, dose-escalation, doubleblind, block-randomized, controlled trial of safety and efficacy of neosaxitoxin alone and in combination with $0.2 \%$ bupivacaine, with and without epinephrine, for cutaneous anesthesia. Anesthesiology 123:873-885.

McCormack K, Santos S, Chapman ML, Krafte DS, Marron BE, West CW, Krambis MJ, Antonio BM, Zellmer SG, Printzenhoff D, et al. (2013) Voltage sensor interaction site for selective small molecule inhibitors of voltage-gated sodium channels. Proc Natl Acad Sci USA 110:E2724-E2732.
Muroi Y and Chanda B (2009) Local anesthetics disrupt energetic coupling between the voltage-sensing segments of a sodium channel. J Gen Physiol 133:1-15.

Nguyen PT, DeMarco KR, Vorobyov I, Clancy CE, and Yarov-Yarovoy V (2019) Structural basis for antiarrhythmic drug interactions with the human cardiac sodium channel. Proc Natl Acad Sci USA 116:2945-2954.

Patton DE and Goldin AL (1991) A voltage-dependent gating transition induces usedependent block by tetrodotoxin of rat IIA sodium channels expressed in Xenopus oocytes. Neuron 7:637-647.

Pero JE, Rossi MA, Lehman HDGF, Kelly MJ III, Mulhearn JJ, Wolkenberg SE, Cato MJ, Clements MK, Daley CJ, Filzen T, et al. (2017) Benzoxazolinone aryl sulfonamides as potent, selective $\mathrm{Na}_{\mathrm{v}} 1.7$ inhibitors with in vivo efficacy in a preclinical pain model. Bioorg Med Chem Lett 27:2683-2688.

Ragsdale DS, McPhee JC, Scheuer T, and Catterall WA (1994) Molecular determinants of state-dependent block of $\mathrm{Na}+$ channels by local anesthetics. Science $\mathbf{2 6 5}$ : $1724-1728$

Ragsdale DS, McPhee JC, Scheuer T, and Catterall WA (1996) Common molecular determinants of local anesthetic, antiarrhythmic, and anticonvulsant block of voltage-gated $\mathrm{Na}^{+}$channels. Proc Natl Acad Sci USA 93:9270-9275.

Sheets MF and Hanck DA (2007) Outward stabilization of the S4 segments in domains III and IV enhances lidocaine block of sodium channels. J Physiol 582: 317-334.

Sheets PL, Jarecki BW, and Cummins TR (2011) Lidocaine reduces the transition to slow inactivation in $\mathrm{Na}(\mathrm{v}) 1.7$ voltage-gated sodium channels. $\mathrm{Br} J$ Pharmacol 164 $719-730$.

Sun S, Jia Q, Zenova AY, Wilson MS, Chowdhury S, Focken T, Li J, Decker S, Grimwood ME, Andrez JC, et al. (2019) Identification of selective acyl sulfonamidecycloalkylether inhibitors of the voltage-gated sodium channel $\left(\mathrm{Na}_{\mathrm{v}}\right) 1.7$ with potent analgesic activity. $J$ Med Chem 62:908-927.

Swain NA, Batchelor D, Beaudoin S, Bechle BM, Bradley PA, Brown AD, Brown B Butcher KJ, Butt RP, Chapman ML, et al. (2017) Discovery of clinical candidate 4 [2-(5-amino-1h-pyrazol-4-yl)-4-chlorophenoxy]-5-chloro-2-fluoro-n-1,3-thiazol-4ylbenzenesulfonamide (pf-05089771): design and optimization of diaryl ether aryl sulfonamides as selective inhibitors of Na v 1.7. J Med Chem 60:7029-7042.

Templin JS, Wylie MC, Kim JD, Kurgansky KE, Gorski G, Kheir J, Zurakowski D, Corfas G, and Berde C (2015) Neosaxitoxin in rat sciatic block: improved therapeutic index using combinations with bupivacaine, with and without epinephrine. Anesthesiology 123:886-898.

Theile JW, Fuller MD, and Chapman ML (2016) The selective Nav1.7 inhibitor, PF05089771 , interacts equivalently with fast and slow inactivated Nav1.7 channels. Mol Pharmacol 90:540-548.

Vedantham V and Cannon SC (1999) The position of the fast-inactivation gate during lidocaine block of voltage-gated Na+ channels. J Gen Physiol 113:7-16.

Wang SY, Mitchell J, Moczydlowski E, and Wang GK (2004) Block of inactivationdeficient $\mathrm{Na}+$ channels by local anesthetics in stably transfected mammalian cells: evidence for drug binding along the activation pathway. J Gen Physiol 124: 691-701.

Wang GK and Wang SY (2014) Block of human cardiac sodium channels by lacosamide: evidence for slow drug binding along the activation pathway. Mol Pharmacol 85:692-702.

Wu YJ, Guernon J, McClure A, Luo G, Rajamani R, Ng A, Easton A, Newton A, Bourin C, Parker D, et al. (2017) Discovery of non-zwitterionic aryl sulfonamides as $\mathrm{Na}_{\mathrm{v}} 1.7$ inhibitors with efficacy in preclinical behavioral models and translational measures of nociceptive neuron activation. Bioorg Med Chem $\mathbf{2 5}$ : 5490-5505.

Wu YJ, Guernon J, McClure A, Venables B, Rajamani R, Robbins KJ, Knox RJ, Matchett M, Pieschl RL, Herrington J, et al. (2018) Discovery of morpholine-based aryl sulfonamides as $\mathrm{Na}_{\mathrm{v}} 1.7$ inhibitors. Bioorg Med Chem Lett 28:958-962.

Yarov-Yarovoy V, Brown J, Sharp EM, Clare JJ, Scheuer T, and Catterall WA (2001) Molecular determinants of voltage-dependent gating and binding of pore-blocking drugs in transmembrane segment IIIS6 of the $\mathrm{Na}(+)$ channel alpha subunit. J Bio Chem 276:20-27.

Yarov-Yarovoy V, McPhee JC, Idsvoog D, Pate C, Scheuer T, and Catterall WA (2002) Role of amino acid residues in transmembrane segments IS6 and IIS6 of the Na+ channel alpha subunit in voltage-dependent gating and drug block. $J$ Biol Chem 277:35393-35401.

Address correspondence to: Bruce P. Bean, Department of Neurobiology, Harvard Medical School, 220 Longwood Ave, Boston MA 02115. E-mail: bruce_bean@hms.harvard.edu 